\title{
A Perturbation Technique to Analyze the Influence of Fiber Weave Effects on Differential Signaling
}

\author{
Mykola Chernobryvko, Dries Vande Ginste and Daniël De Zutter \\ Electromagnetics Group, Dept. of Information Technology, Ghent University, Gent, Belgium \\ Email: mykola.chernobryvko@intec.ugent.be
}

\begin{abstract}
We study differential signaling via a pair of striplines in a substrate that is comprised of an epoxy/fiberglass woven composite structure. The transmission characteristics, which are deteriorated due to the presence of the fiber weave, are analyzed via an efficient modeling technique for nonuniform transmission lines. This technique is based on the solution of the pertinent differential equations using a perturbation approach. For a challenging application example, it is shown that the unavoidable phase errors can be controlled by subdividing electrically long lines into smaller pieces, as such increasing accuracy whilst maintaining efficiency.
\end{abstract}

Keywords-Nonuniform transmission line, perturbation, fiber weave, mode conversion

\section{INTRODUCTION}

Nonuniform transmission lines (NUTLs) are found in various applications, such as filters, impedance transformers, etc. The modeling of these interconnects has, however, always been a challenging problem. Due to the varying per-unitlength (p.u.l.) parameters along the NUTL, the differential equations describing them cannot be solved analytically, except for some very special cases. In [1], the authors of the present contribution presented a two-step perturbation approach to model single and differential pairs of NUTLs. The focus was on the theoretical description of the technique and on demonstrating its applicability and limitations. In this contribution, however, we extend the perturbation technique [1] in order to apply it to a very important, but challenging, application example. Leveraging the novel perturbation technique, we analyze differential signaling using a pair of striplines embedded in a substrate that is comprised of an epoxy/fiberglass woven composite structure [2], [3]. In such a commonly used substrate, it is very likely that one trace of the differential pair is located mainly in the epoxy resin with low dielectric constant, while the other trace is located close to the glass fiber with a high dielectric constant. As the two traces "see" a different permittivity, a differential skew between the lines is observed. This skew results in insertion loss suck-outs of the transmitted (differential) signal. Additionally, the imbalance leads to conversion from the differential mode to the common mode [4]. All this may prohibit the use of these substrates at very high frequencies, or differently put, it poses a limit on the maximum (electrical) length of the lines.

\section{PERTURbATION SOLUTION FOR A DIFFERENTIAL LINE PAIR}

We analyse nonuniform differential lines within the framework of the quasi-TM approach and in the frequency domain (with the $e^{j \omega t}$ dependency suppressed). Consider voltage and current column vectors $\mathcal{V}=\left[V_{1} V_{2}\right]^{T}$ and $\mathcal{I}=\left[I_{1} I_{2}\right]^{T}$, holding the two voltages and two currents along the lines. To simplify the notations we work with $2 \times 2$ complex p.u.l. inductance $\mathcal{L}$ and capacitance $\mathcal{C}$ matrices, i.e. the p.u.l. resistance $\mathcal{R}$ and conductance $\mathcal{G}$ are understood to be part of $\mathcal{L}$ and $\mathcal{C}$ $\left(\mathcal{L}=\mathcal{L}+\frac{\mathcal{R}}{j \omega}\right.$ and $\left.\mathcal{C}=\mathcal{C}+\frac{\mathcal{G}}{j \omega}\right)$. Our starting point is the well-known Telegrapher's equations:

$$
\begin{aligned}
& \frac{d \mathcal{V}(z)}{d z}=-j \omega \mathcal{L}(z) \mathcal{I}(z), \\
& \frac{d \mathcal{I}(z)}{d z}=-j \omega \mathcal{C}(z) \mathcal{V}(z),
\end{aligned}
$$

with $z$ being the signal propagation direction. To perform a perturbation technique, the following expansions are introduced:

$$
\begin{aligned}
& \mathcal{V}(z)=\tilde{\mathcal{V}}(z)+\Delta \mathcal{V}_{1}(z)+\Delta \mathcal{V}_{2}(z)+\ldots, \\
& \mathcal{I}(z)=\tilde{\mathcal{I}}(z)+\Delta \mathcal{I}_{1}(z)+\Delta \mathcal{I}_{2}(z)+\ldots, \\
& \mathcal{C}(z)=\tilde{\mathcal{C}}+\Delta \mathcal{C}(z) \\
& \mathcal{L}(z)=\tilde{\mathcal{L}}+\Delta \mathcal{L}(z)
\end{aligned}
$$

The leading terms of the series expansions (3), i.e. the voltage $\tilde{\mathcal{V}}(z)$ and current $\tilde{\mathcal{I}}(z)$, are labeled as the unperturbed values. The remaining terms are perturbations of order one, two, etc. $\mathcal{C}(z)$ and $\mathcal{L}(z)$ in (3) are simply written as the sum of a constant part and a place-dependent part. Here, $\tilde{\mathcal{C}}$ and $\tilde{\mathcal{L}}$ are the unperturbed values. $\Delta \mathcal{C}(z)$ and $\Delta \mathcal{L}(z)$ are the variations of the capacitance and inductance along the line which remain after subtracting the constant martrices $\tilde{\mathcal{C}}$ and $\tilde{\mathcal{L}}$ from $\mathcal{C}(z)$ and $\mathcal{L}(z)$ respectively. Remark that $\tilde{\mathcal{C}}$ and $\tilde{\mathcal{L}}$ are not necessarily the mean values of $\mathcal{C}$ and $\mathcal{L}$ over the line. We only suppose that $\Delta \mathcal{C}(z)$ and $\Delta \mathcal{L}(z)$ are small enough with respect to $\tilde{\mathcal{C}}$ and $\tilde{\mathcal{L}}$. The unperturbed matrices can be written as:

$$
\tilde{\mathcal{C}}=\left(\begin{array}{cc}
C_{a} & -C_{b} \\
-C_{b} & C_{a}
\end{array}\right) \quad \tilde{\mathcal{L}}=\left(\begin{array}{ll}
L_{a} & L_{b} \\
L_{b} & L_{a}
\end{array}\right)
$$

Consequently, the unperturbed solution consists of an even and an odd mode contribution, i.e.:

$$
\begin{aligned}
\tilde{V}_{1}(z) & =\left[\tilde{V}_{e}(z)+\tilde{V}_{o}(z)\right] / 2, \\
\tilde{V}_{2}(z) & =\left[\tilde{V}_{e}(z)-\tilde{V}_{o}(z)\right] / 2, \\
\tilde{I}_{1}(z) & =\left[\tilde{I}_{e}(z)+\tilde{I}_{o}(z)\right] / 2, \\
\tilde{I}_{2}(z) & =\left[\tilde{I}_{e}(z)-\tilde{I}_{o}(z)\right] / 2 .
\end{aligned}
$$


Using (5), the unperturbed differential equations for the even and odd mode are easily found to be

$$
\begin{aligned}
& \frac{d \tilde{V}_{e}(z)}{d z}=-j \omega\left(L_{a}+L_{b}\right) \tilde{I}_{e}(z), \\
& \frac{d \tilde{I}_{e}(z)}{d z}=-j \omega\left(C_{a}-C_{b}\right) \tilde{V}_{e}(z), \\
& \frac{d \tilde{V}_{o}(z)}{d z}=-j \omega\left(L_{a}-L_{b}\right) \tilde{I}_{o}(z), \\
& \frac{d \tilde{I}_{o}(z)}{d z}=-j \omega\left(C_{a}+C_{b}\right) \tilde{V}_{o}(z) .
\end{aligned}
$$

Hence, the modal voltages become:

$$
\begin{aligned}
& \tilde{V}_{e}=\left(A_{1} e^{-j k_{e} z}+B_{1} e^{+j k_{e} z}\right), \\
& \tilde{V}_{o}=\left(A_{2} e^{-j k_{o} z}+B_{2} e^{+j k_{o} z}\right) .
\end{aligned}
$$

Even and odd mode wave numbers $k_{e}$ and $k_{o}$ are given by:

$$
\frac{k_{e}}{\omega}=\sqrt{\left(L_{a}+L_{b}\right)\left(C_{a}-C_{b}\right)}, \frac{k_{o}}{\omega}=\sqrt{\left(L_{a}-L_{b}\right)\left(C_{a}+C_{b}\right)} .
$$

The corresponding modal currents are

$$
\begin{aligned}
& \tilde{I}_{e}=\left(A_{e} e^{-j k_{e} z}-B_{e} e^{+j k_{e} z}\right) / Z_{e} \\
& \tilde{I}_{o}=\left(A_{o} e^{-j k_{o} z}-B_{o} e^{+j k_{o} z}\right) / Z_{o}
\end{aligned}
$$

with the even and odd mode impedances given by

$$
Z_{e}=\sqrt{\frac{L_{a}+L_{b}}{C_{a}-C_{b}}}, \quad Z_{o}=\sqrt{\frac{L_{a}-L_{b}}{C_{a}+C_{b}}} .
$$

The unknown coefficients $A_{e}, A_{o}, B_{e}$ and $B_{o}$ are determined by enforcing the boundary conditions at $z=0$ and $z=l$ in terms of even and odd mode voltages and currents (see [1] for detailed expressions for these boundary conditions). Before turning to the first-order perturbation, let us take a closer look at $\Delta \mathcal{C}$ and $\Delta \mathcal{L} . \Delta \mathcal{C}$ can be written as

$$
\Delta \mathcal{C}=\left(\begin{array}{cc}
\Delta C_{a 1} & -\Delta C_{b} \\
-\Delta C_{b} & \Delta C_{a 2}
\end{array}\right)
$$

As $\tilde{\mathcal{C}}+\Delta \mathcal{C}$ must have all the properties of a proper capacitance matrix in each point along the line pair, it can be asserted that the above matrix is symmetric but the entries of the matrix can either be positive or negative. It is useful to rewrite (11) as:

$$
\begin{aligned}
\Delta \mathcal{C} & =\left(\begin{array}{cc}
\frac{\Delta C_{a 1}+\Delta C_{a 2}}{2} & -\Delta C_{b} \\
-\Delta C_{b} & \frac{\Delta C_{a 1}+\Delta C_{a 2}}{2}
\end{array}\right) \\
+ & \left(\begin{array}{cc}
\frac{\Delta C_{a 1}-\Delta C_{a 2}}{2} & 0 \\
0 & -\frac{\Delta C_{a 1}-\Delta C_{a 2}}{2}
\end{array}\right)
\end{aligned}
$$

and

$$
\begin{aligned}
\Delta \mathcal{L} & =\left(\begin{array}{cc}
\frac{\Delta L_{a 1}+\Delta L_{a 2}}{2} & \Delta L_{b} \\
\Delta L_{b} & \frac{\Delta L_{a 1}+\Delta L_{a 2}}{2}
\end{array}\right) \\
+ & \left(\begin{array}{cc}
\frac{\Delta L_{a 1}-\Delta L_{a 2}}{2} & 0 \\
0 & -\frac{\Delta L_{a 1}-\Delta L_{a 2}}{2}
\end{array}\right) .
\end{aligned}
$$

With (12) and (13), the differential equations for the even and odd mode first-order perturbation, become

$$
\begin{aligned}
& \frac{d \Delta V_{1 e}}{d z}=-j \omega\left(L_{a}+L_{b}\right) \Delta I_{1 e}-j \omega\left(l_{a}+l_{b}\right) \tilde{I}_{e}-j \omega l \tilde{I}_{o}, \\
& \frac{d \Delta I_{1 e}}{d z}=-j \omega\left(C_{a}-C_{b}\right) \Delta V_{1 e}-j \omega\left(c_{a}-c_{b}\right) \tilde{V}_{e}-j \omega c \tilde{V}_{o}, \\
& \frac{d \Delta V_{1 o}}{d z}=-j \omega\left(L_{a}-L_{b}\right) \Delta I_{1 o}-j \omega\left(l_{a}-l_{b}\right) \tilde{I}_{o}-j \omega l \tilde{I}_{e}, \\
& \frac{d \Delta I_{1 o}}{d z}=-j \omega\left(C_{a}+C_{b}\right) \Delta V_{1 o}-j \omega\left(c_{a}+c_{b}\right) \tilde{V}_{o}-j \omega c \tilde{V}_{e}
\end{aligned}
$$

with

$$
\begin{array}{ccc}
c_{a}=\frac{\Delta C_{a 1}+\Delta C_{a 2}}{2}, & c_{b}=\Delta C_{b}, & c=\frac{\Delta C_{a 1}-\Delta C_{a 2}}{2} \\
l_{a}=\frac{\Delta L_{a 1}+\Delta L_{a 2}}{2}, & l_{b}=\Delta L_{b}, & l=\frac{\Delta L_{a 1}-\Delta L_{a 2}}{2}
\end{array}
$$

In (14) we have a separate set of equations for the two modes: the even mode comes with the $\left(C_{a}-C_{b}, L_{a}+L_{b}\right)$ p.u.l. set; the odd mode with the $\left(C_{a}+C_{b}, L_{a}-L_{b}\right)$ p.u.l. set. These equations still looks like Telegrapher's equations, but now, with additional distributed source terms. The source terms are responsible for mode coupling. By rewriting $\Delta \mathcal{C}$ and $\Delta \mathcal{L}$ as in (12) and (13), it becomes clear which part of the variation of the capacitance and inductance along the line is responsible for perturbation with and without mode coupling. The solution of (14) can be derived with standard mathematical techniques, as described in [1] for the single line case, but now for evenand odd-mode voltages and currents.

Following the same procedure as just outlined above, a second perturbation step may now be introduced, yielding equations similar to (14). As shown in [1], this second perturbation leads to a substantial gain in accuracy.

\section{FIBER WEAVE APPLICATION EXAMPLE}

\section{A. Description of the example}

To demonstrate our technique, consider the transmission of a differential signal over two copper $\left(\sigma=5.8 \cdot 10^{7} \mathrm{~S} / \mathrm{m}\right)$ stripline tracks embedded in a substrate. This substrate is nonhomogeneous due to the presence of fiber weave, as detailed below. The stripline pair is depicted in Fig. 1. The conductor thickness is $35 \mu \mathrm{m}$. The tracks are $180 \mu \mathrm{m}$ wide with a distance of $630 \mu \mathrm{m}$ separating them. The distance between top and bottom plate is $420 \mu \mathrm{m}$. These dimensions are such that at $10 \mathrm{GHz}$, a single line has an impedance of $50 \Omega$ when a homogeneous background medium with $\varepsilon_{r}=3.4$ is considered. However, here we consider a type 1080 fiber weave substrate, the top view of which is depicted in Fig. 2. To clearly illustrate the effect of fiber weave, we have opted to put the left line (line 1) on top of a glass bundle while the right line (line 2) mainly "sees" epoxy prepreg. Consequently, the tracks - which are running in the warp direction - are embedded in a periodically changing background medium. To model this background medium we consider two different crosssections, indicated as cross-sections $a$ and $b$ in Fig. 2. These two cross-sections and all relevant dimensions are detailed in Figs. 3 and 4 respectively. The dielectric constant and the loss tangent of the glass and the epoxy prepreg are described by 


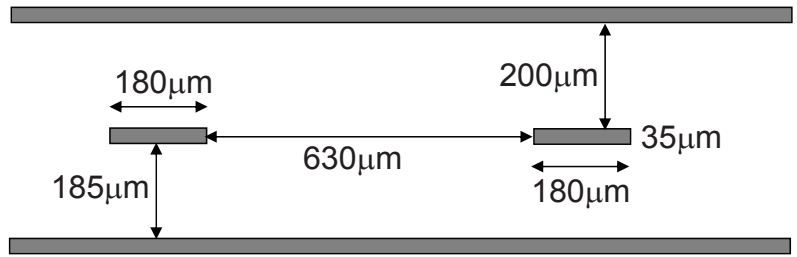

Fig. 1. Geometry of the differential stripline pair.

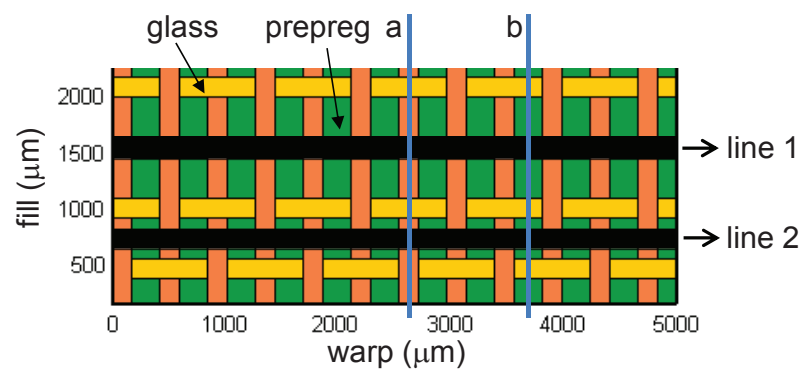

Fig. 2. Top view of the positioning of the lines w.r.t. the fiber weave.

a Debye model. For the glass, Fig. 5 depicts the real part of the relative dielectric constant, i.e. $\varepsilon_{r}^{\prime}$, and the loss tangent, $\tan \delta$, as a function of frequency. At $1 \mathrm{GHz}, \varepsilon_{r}^{\prime}=6$ and $\tan \delta=0.015$. For the epoxy a similar model is used with the same loss tangent but with $\varepsilon_{r}^{\prime}=3$ at $1 \mathrm{GHz}$. The RLGCparameters in each cross-section are modeled using an integral equation for the equivalent polarization charges and for the equivalent differential surface currents [5].

In the propagation direction $z$, the stripline pair is now modeled as the concatenation of alternating sections $a$ and $b$, i.e. $a-b-a-b-\ldots$ Section $a$ has a length of $171 \mu \mathrm{m}$; section $b$ has a length of $253 \mu \mathrm{m}$. In this contribution we investigate such a line with a total length of $25.4 \mathrm{~cm}=10^{\prime \prime}$, i.e. sections $a$ and $b$ are alternately repeated 600 times.

\section{B. Numerical results}

Such a model, where the p.u.l. parameters vary in a piecewise constant manner, allows using the chain matrix approach [6] as a reference technique. In this approach, the chain matrices of sections $a$ and $b$ are computed and the overall result is obtained by alternately concatenating the sections, i.e. by multiplication of the $2 \times 600=1200$ chain matrices.

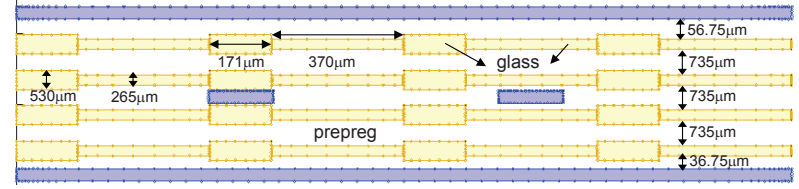

Fig. 3. Detail of cross-section $a$ as defined in Fig. 2.

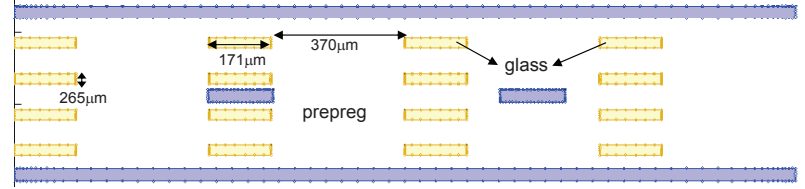

Fig. 4. Detail of cross-section $b$ as defined in Fig. 2.

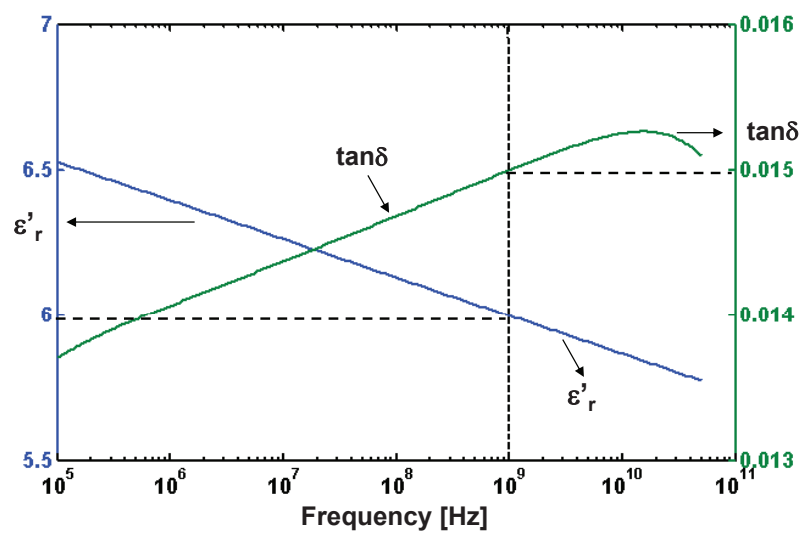

Fig. 5. Variation of the real part of the relative dielectric permittivity (left) and the loss tangent (right) of the fiber weave glass as a function of frequency.

In contrast to the chain matrix approach, the perturbation technique proposed in this contribution can also handle continuously varying p.u.l. parameters. Due care has to be taken, however, to ensure a high precision for electrically (very) long lines. Indeed, as reported in [1], when the line becomes electrically long, say about five to ten wavelengths, phase errors start to accumulate. Unfortunately, the fiber weave effect as described in the introduction, is best visible for very long lines, such as the one we analyze here. (Indeed, assuming an average $\epsilon_{r}$ of 3.4, a line length of $10^{\prime \prime}$ corresponds to approximately 80 wavelengths at $50 \mathrm{GHz}$ !) To improve the accuracy of the method at very high frequencies, we need to subdivide the long line into shorter sections, model these sections separately with the perturbation technique and concatenate the models. Nonetheless, the number of sections may remain limited, making the perturbation technique still much faster than the reference chain matrix technique, as shown below.

First, we focus on the accuracy of the perturbation technique by presenting mixed-mode $S$-parameters w.r.t. $50 \Omega$ reference impedances [4]. In particular, we study the magnitude of the differential transmission coefficient $S_{d d 21}$ and the differential-to-common-mode conversion $S_{c d 21}$ in the frequency range from $\mathrm{DC}$ to $50 \mathrm{GHz}$, as shown in Figs. 6(a) and (b). To perform the perturbation analysis up to $50 \mathrm{GHz}$, the line was subdivided into 20 sections. As can be seen, the results of the perturbation approach are very accurate in comparison to the reference technique. To assess the influence of the fiber weave, the results for a uniform interconnect with constant (unperturbed) p.u.l. $C$ - and $L$-matrices as specified in (4), are also shown. It is clear that, next to mode conversion, the presence of fiber weave leads to insertion loss suck-outs. Additionally, Fig. 7 is shown to demonstrate the evolution of the accuracy of the perturbation technique when we vary the number of concatenated sections. When modeling the entire line of $10^{\prime \prime}$ as one section, the perturbation technique captures the correct behavior accurately up to $5 \mathrm{GHz}$ (ca. 8 wavelengths). Modeling five sections of $2^{\prime \prime}$ each, allows to improve the results up to the first insertion loss suck-out peak at ca. $13 \mathrm{GHz}$. A section of $1^{\prime \prime}$ can be accurately modeled up to $35 \mathrm{GHz}$ and to obtain accurate results up to $50 \mathrm{GHz}, 20$ sections are needed, as was already presented in Fig. 6(a). 


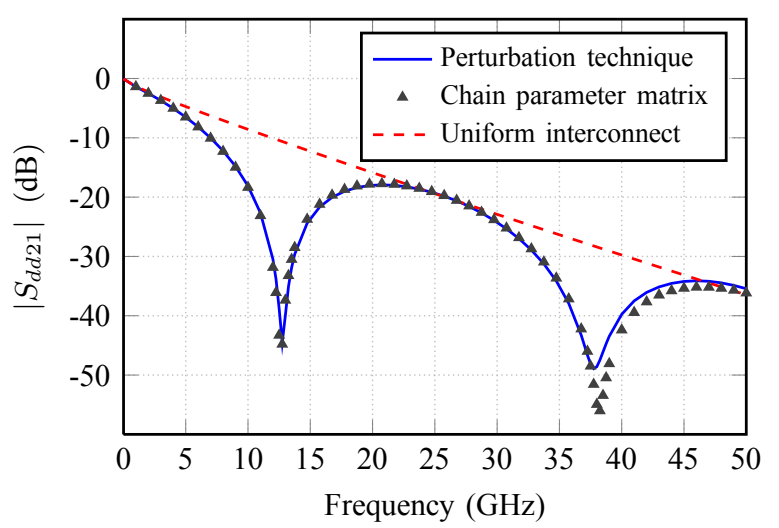

(a)

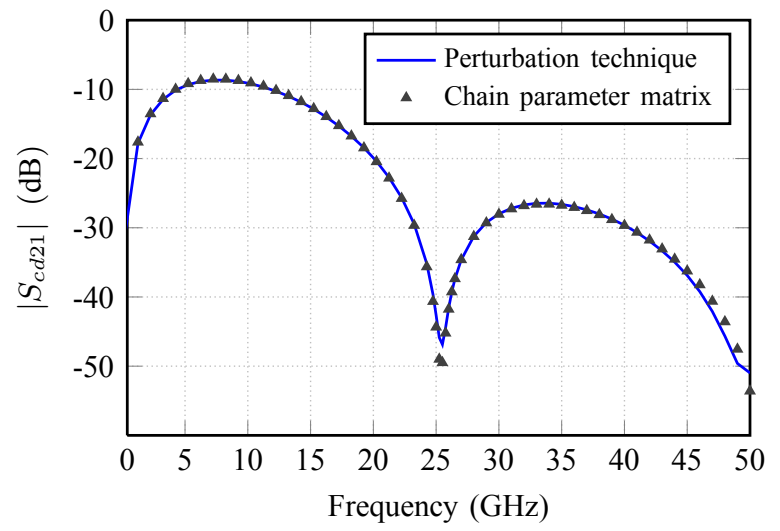

(b)

Fig. 6. (a) Differential mode transmission coefficient and (b) forward differential-to-common mode conversion of the pair of coupled lines embedded in the fiber weave substrate. For the perturbation technique, the line was divided into 20 sections that were modeled separately and then concatenated. The chain matrix approach relies on a concatenation of all 1200 sections. To illustrate the fiber weave effect, the differential mode transmission coefficient for a uniform interconnect is also shown.

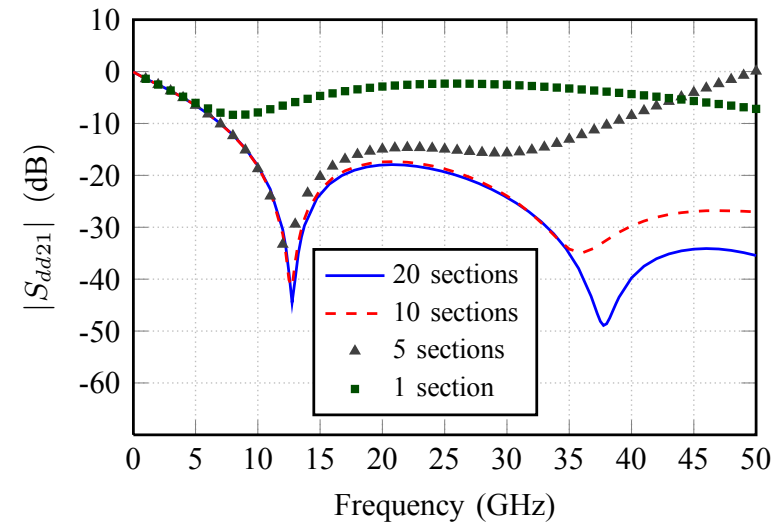

Fig. 7. Differential mode transmission coefficient of the entire line obtained with the perturbation technique when subdividing the line into a varying number of sections.

TABLE I. EFFICIENCY OF THE PERTURBATION TECHNIQUE.

THE CPU TIME NEEDED WITH THE REFERENCE APPROACH IS $156.3 \mathrm{~S}$.

\begin{tabular}{|c||c|c|}
\hline $\begin{array}{c}\text { Number of } \\
\text { sections }\end{array}$ & $\begin{array}{c}\text { CPU } \\
\text { time }\end{array}$ & $\begin{array}{c}\text { Speed-up } \\
\text { factor }\end{array}$ \\
\hline \hline 1 & $10.6 \mathrm{~s}$ & 14.7 \\
\hline 5 & $15.8 \mathrm{~s}$ & 9.9 \\
\hline 10 & $22.8 \mathrm{~s}$ & 6.9 \\
\hline 20 & $35.6 \mathrm{~s}$ & 4.4 \\
\hline
\end{tabular}

Second, to demonstrate the efficiency of our novel technique, we consider the computation time of the code in Matlab R2009a. All calculations were performed on a computer with an Intel(R) Core(TM) Quad CPU Q9650 and 8 GB of installed memory (RAM) for 201 frequency samples (linearly spaced between DC and $50 \mathrm{GHz}$ ). Table I shows the computation time of the perturbation method for a varying number of sections. The speed-up factor is calculated w.r.t. the CPU time of $156.3 \mathrm{~s}$ needed by the reference technique. As can be seen, even when subdividing the line into 20 sections, we still obtain a speed-up factor of 4.4 .

\section{CONCLUSION}

A perturbation technique to model NUTLs, in particular differential lines embedded in a substrate composed of woven glass fibers, was presented. The fiber weave effect causes a differential skew between the two traces, leading to insertion loss suck-outs and mode conversion. By subdividing electrically very long lines into a limited number of shorter sections of about 5 to 10 wavelengths, these fiber weave effects are precisely captured by the perturbation technique. Compared to a standard chain matrix approach, excellent accuracy and improved efficiency was obtained. Additionally, it is worth mentioning that the reference chain matrix approach does not allow the modeling of NUTLs with continuously varying p.u.l. parameters. Such NUTLs will however be further investigated, leveraging the novel perturbation technique.

\section{ACKNOWLEDGMENT}

The authors like to thank Stefaan Sercu and Jan De Geest of FCI for providing a model of the fiber weave cross-sections.

\section{REFERENCES}

[1] M. Chernobryvko, D. Vande Ginste, and D. De Zutter, "A two-step perturbation technique for nonuniform single and differential lines," IEEE Trans. Microw. Theory Tech., vol. 61, no. 5, pp. 1758 - 1767, May 2013.

[2] S. McMorrow and C. Heard, "The impact of PCB laminate weave on the electrical performance of differential signaling at multi-gigabit data rates," in Proc. DesignCon, 2005.

[3] E.-P. Li, X.-C. Wei, A. C. Cangellaris, E.-X. Liu, Y.-J. Zhang, M. D'Amore, J. Kim, and T. Sudo, "Progress review of electromagnetic compatibility analysis technologies for packages, printed circuit boards, and novel interconnects," IEEE Trans. Electromagn. Compat., vol. 52, no. 2, pp. 248-265, May 2010.

[4] C. Gazda, D. Vande Ginste, H. Rogier, R.-B. Wu, and D. De Zutter, "A wideband common-mode suppression filter for bend discontinuities in differential signaling using tightly coupled microstrips," IEEE Trans. Adv. Packag., vol. 33, no. 4, pp. 969-978, Nov. 2010.

[5] T. Demeester and D. De Zutter, "Quasi-TM transmission line parameters of coupled lossy lines based on the Dirichlet to Neumann boundary operator," IEEE Trans. Microw. Theory Tech., vol. 56, no. 7, pp. 16491660, Jul. 2008.

[6] C. R. Paul, Analysis of Multiconductor Transmission Lines. John Wiley \& Sons, 1994. 